\title{
LIM kinase 1 serves an important role in the multidrug resistance of osteosarcoma cells
}

\author{
JIAN-ZENG YANG ${ }^{1,2}$, LI-HONG HUANG ${ }^{3}$, RUI CHEN ${ }^{1}$, LING-JIE MENG ${ }^{1}$, \\ YI-YAO GAO ${ }^{1}$, QIU-YE JI ${ }^{1}$ and YAN WANG ${ }^{1}$ \\ ${ }^{1}$ Scientific Research Center, China-Japan Union Hospital of Jilin University, Changchun, Jilin 130033; \\ ${ }^{2}$ Department of Nuclear Medicine, First Affiliated Hospital of Zhengzhou University, Zhengzhou, Henan 450052; \\ ${ }^{3}$ Department of Physiology and Medicine, China-Japan Union Hospital of Jilin University, Changchun, Jilin 130033, P.R. China
}

Received December 24, 2015; Accepted April 21, 2017

DOI: 10.3892/ol.2017.7317

\begin{abstract}
Multidrug resistance (MDR) is a major challenge for the management of the majority of cancers. The precise molecular mechanisms of MDR remain elusive. In a previous study, a multidrug resistant osteosarcoma model [MG63/vincristine (VCR)] was established by intermittent exposure of MG63 cells to gradually increasing concentrations of VCR. These cells exhibited cross-resistance to multiple structurally and mechanistically unrelated chemotherapeutic agents. The development of MDR was associated with increased expression of LIM kinase 1 (LIMK1). Compared with that in normal human fetal osteoblasts (hFOB) 1.19, the messenger RNA and protein expression of LIMK1 was significantly elevated both in MG63 and U2OS osteosarcoma cells. To observe the expression pattern of LIMK1 in osteosarcoma, immunohistochemical analyses were performed on specimens derived from 6 patients. The results indicated that LIMK1 was expressed to a greater extent in the tumor parenchyma than in the mesenchyme. The role of LIMK1 in MDR was confirmed by transfecting plasmids coding LIMK1-small interfering RNA (siRNA), wild-type-LIMK1 or empty vector into MG63/VCR cells, and measuring the expression of LIMK1 and multidrug resistance protein 1 (MDR1), also known as P-gycoprotein (P-gp). The results demonstrated that the level of MDR1/P-gp was positively correlated with the level of
\end{abstract}

Correspondence to: Professor Yan Wang, Scientific Research Center, China-Japan Union Hospital of Jilin University, 126 Xiantai Street, Changchun, Jilin 130033, P.R. China

E-mail: wangy01@jlu.edu.cn

Abbreviations: CCK-8, Cell Counting kit-8; DOX, doxorubicin; H-DMEM, high glucose-Dulbecco's modified Eagle's medium; hFOB, human fetal osteoblasts; LIMK1, LIM kinase 1; MDR, multidrug resistance; P-gp, P-glycoprotein; PI, propidium iodide; MTX, methotrexate; PTX, paclitaxel; THP, pirarubicin; VCR, vincristine

Key words: osteosarcoma, LIMK1, immunochemistry, multidrug resistance
LIMK1. This correlation was also shown with the doxorubicin efflux assay and by measuring apoptosis. Specifically, after $6 \mathrm{~h}$ of incubation with VCR, $25.6 \%$ of the cells transfected with the LIMK1-siRNA plasmid were apoptotic compared with $6.2 \%$ in the empty vector group and $1.3 \%$ in the group of cells transfected with the wild-type-LIMK1 plasmid. Thus, it was concluded that LIMK1 serves a key role in the MDR of osteosarcoma and functions through MDR1.

\section{Introduction}

Osteosarcoma is a highly malignant bone tumor with widespread histological heterogeneity, a lack of biomarkers, high local aggressiveness and a rapid metastasizing potential (1). Osteosarcoma is the most common primary bone malignancy and the third most common cancer in adolescents and young adults (2). Chemotherapy combined with surgery is the principal mode of treatment for osteosarcoma. However, tumor resistance to chemotherapy and molecularly targeted therapies limits their effectiveness $(1,3)$. Tumor cells may become cross-resistant to a broad spectrum of chemotherapeutic agents with different mechanisms and structures following a single-drug treatment; this is known as multidrug resistance (MDR) (4). Within tumor cells, various MDR mechanisms can operate, including increased drug efflux, mutations of the drug target, enhanced repair of DNA damage, activation of alternative signaling pathways and evasion of cell death (3). Although the precise mechanisms of MDR remain unclear, several cell membrane transporter proteins are linked to the resistance observed with commonly used chemotherapeutic agents. For example, multidrug resistance protein 1 (MDR1), also known as P-glycoprotein (P-gp), belongs to the ATP-binding cassette (ABC) transporter family, and regulates the efflux of multiple structurally and mechanistically unrelated chemotherapeutic agents across the plasma membrane (3).

LIM kinase 1 (LIMK1) is a member of the serine/threonine protein kinase family. It is activated when the Thr508 residue is phosphorylated by Rho-GTPases such as Rho, Rac and cell division cycle $42(5,6)$. LIMK1 functions through regulating actin cytoskeleton remodeling by phosphorylating and inactivating cofilin on the Ser3 residue $(7,8)$. Increasing evidence suggests that LIMK1 serves an important role in 
tumor metastasis and invasion (9). However, few studies have addressed the role of LIMK1 in MDR in tumor cells (10). In a previous study, an osteosarcoma MDR model was established by using the human osteosarcoma cell line MG63 and vincristine (VCR), a drug commonly used in the treatment of osteosarcoma (11). Notably, the development of MDR was associated with increased expression of LIMK1. Therefore, the current study was designed to determine whether the overexpression of LIMK1 contributes to MDR and to identify its potential mechanism.

\section{Materials and methods}

Cells and cell culture. The human osteosarcoma cell lines U2OS and MG63 (purchased from the Institute of Biochemistry and Cell Biology, Chinese Academy of Sciences, Shanghai, China) were cultured in high glucose-Dulbecco's modified Eagle's medium (H-DMEM; Gibco; Thermo Fisher Scientific, Inc., Waltham, MA, USA) containing $10 \%$ fetal bovine serum (FBS; Hyclone, Logan, UT, USA). Human fetal osteoblastic (hFOB) 1.19 cells (donated by the Pathology Laboratory of Jilin University, Changchun, China) were cultured in DMEM-F12 (Gibco; Thermo Fisher Scientific, Inc.) supplemented with $10 \%$ FCS. All three cell types were incubated at $37^{\circ} \mathrm{C}$ in a humidified atmosphere containing $5 \% \mathrm{CO}_{2}$. Tumor samples from 6 patients with osteosarcoma were collected intra-operatively and stored at $-80^{\circ} \mathrm{C}$ (March 2015 form the China-Japan Union Hospital of Jilan University, Changchun, China). Written consent was obtained from all patients or their families, and the study was conducted in accordance with the Declaration of Helsinki. The protocol was approved by the Institutional Review Board of Jilin University.

Establishment of VCR-resistant cell sublines. To generate VCR-resistant sublines, MG63 cells were exposed to increasing concentrations of VCR as described previously (11). Briefly, parental MG63 cells were cultured initially in H-DMEM containing $10 \mathrm{ng} / \mathrm{ml}$ VCR (Shanghai New Hualian Pharmaceutical Co., Ltd., Shanghai, China) for 72 h. Surviving cells were collected and cultured in VCR-free medium for 1-2 weeks, and then further cultured with VCR-containing H-DMEM. This procedure was repeated $\geq 5$ times until the majority of cells survived in the drug-containing medium. Cells were then exposed to increasing concentrations $(10-500 \mathrm{ng} / \mathrm{ml})$ of VCR following the same procedure. This process required 4-6 weeks to establish adequate growth at each VCR concentration. Eventually, a subline resistant to $500 \mathrm{ng} / \mathrm{ml}$ VCR was obtained, which was named MG63/VCR. Prior to each experiment, MG63 and MG63/VCR cells were maintained in drug-free media and subcultured $\geq 3$ times.

Drug sensitivity assays. The Cell Counting kit-8 (CCK-8) (Dojindo Molecular Technologies, Inc., Kumamoto, Japan) assay was used to determine the sensitivities of cells to VCR, pirarubicin (THP), methotrexate (MTX), doxorubicin (DOX) (all from Hualian Pharmaceutical Co., Shanghai, China) and paclitaxel (PTX) (Laboratories Pierre Fabre, Castres, Cedex, France). Cells in the logarithmic growth phase were trypsinized and plated onto 96 -well plates at $8 \times 10^{3}$ cells/well, and cultured for $24 \mathrm{~h}$ in $100 \mu \mathrm{l} \mathrm{H}$-DMEM supplemented with $10 \%$
FBS (Hyclone). The culture medium was then replaced with medium containing serial dilutions of each drug $(125,25,5$, $1,0.2,0.04,0.008$ and $0.0016 \mu \mathrm{g} / \mathrm{ml})$. Drug-free medium was added to the control and blank wells. After $48 \mathrm{~h}$, the medium was replaced with $10 \% \mathrm{CCK}-8$. After incubation at $37^{\circ} \mathrm{C}$ for $2 \mathrm{~h}$, the plates were analyzed in a plate reader at $450 \mathrm{~nm}$. The percentage of inhibition for each treatment was calculated using the following formula: Inhibition $=\left(\mathrm{OD}_{\text {control }}{ }^{-} \mathrm{OD}\right.$ experimental group $) / \mathrm{OD}_{\text {control }} \mathrm{x} 100$. Based on this information, the $50 \%$ inhibitory concentration $\left(\mathrm{IC}_{50}\right)$ was calculated using GraphPad Prism 5 software (GraphPad Software, Inc., La Jolla, CA, USA). The resistance index (RI) was calculated as $\mathrm{IC}_{50 \mathrm{MG} 63 / \mathrm{VCR}} / \mathrm{IC}_{50 \mathrm{MG} 63}$.

Immunohistochemical staining. Immunohistochemical staining was performed as described previously (12). Briefly, tumor tissue sections were incubated with $3 \% \mathrm{H}_{2} \mathrm{O}_{2}$ to inactivate endogenous peroxidases, followed by deparaffinization and rehydration. The antigen was released with $3 \%$ proteinase $\mathrm{K}$, followed by washing with PBS and blocking with $10 \%$ goat serum (Santa Cruz Biotechnology, Inc.) for $1 \mathrm{~h}$ at room temperature. The sections were then incubated for $1 \mathrm{~h}$ at room temperature with a mouse monoclonal antibody targeting $\beta$-actin (sc-58673, dilution 1:500; Santa Cruz Biotechnology, Inc., Dallas, TX, USA) or a rabbit polyclonal antibody targeting LIMK1 (sc-48346, dilution 1:100; Santa Cruz Biotechnology, Inc.). Biotinylated rabbit anti-mouse immunoglobulin (sc-2359, Santa Cruz Biotechnology, Inc.) diluted 1:100 in 3\% normal goat serum (Santa Cruz Biotechnology, Inc.) was applied for $1 \mathrm{~h}$ at room temperature, followed by incubation with a horseradish peroxidase (HRP)-conjugated streptavidin-biotin complex (Santa Cruz Biotechnology, Inc.) for $10 \mathrm{~min}$ at room temperature. Paraffin-embedded sections were dehydrated, mounted with neutral gum and observed under a light microscope (Olympus Corporation, Tokyo, Japan).

Immunoprecipitation and western blot analysis. U2OS, MG63 and hFOB1.19 cells were lysed with lysis buffer [150 mM $\mathrm{NaCl}, 1 \%$ Nonidet P-40, $50 \mathrm{mM}$ Tris- $\mathrm{HCl}$ (pH 8.0) containing $20 \mu \mathrm{M}$ phenylmethylsulfonyl fluoride] at $4^{\circ} \mathrm{C}$ for $30 \mathrm{~min}$, and then centrifuged at $12,000 \mathrm{x}$ g at $4^{\circ} \mathrm{C}$ for $15 \mathrm{~min}$. The supernatant was stored at $-70^{\circ} \mathrm{C}$. Equal amounts of protein $(100 \mathrm{ng})$, $15 \mu \mathrm{l}$ of protein A agarose beads (Santa Cruz Biotechnology, Inc.) and $1 \mu \mathrm{l}$ of anti-LIMK1 antibody (sc-48346, Santa Cruz Biotechnology, Inc.) were added to the tubes, which were rotated overnight at $4^{\circ} \mathrm{C}$. The agarose-antibody-antigen complexes were collected by centrifugation for $20 \mathrm{sec}$ at $12,000 \times \mathrm{g}$ and $4^{\circ} \mathrm{C}$. The supernatant was removed carefully, and the complexes were washed twice with lysis buffer. The pellet was resuspended in gel-loading buffer (Santa Cruz Biotechnology, Inc.), and the proteins were denatured by boiling for $5 \mathrm{~min}$. Protein A agarose beads were removed by centrifugation at $12,000 \mathrm{x} \mathrm{g}$ for $20 \mathrm{sec}$ at $4^{\circ} \mathrm{C}$, and the supernatant was transferred to a fresh tube. The proteins (20 $\mu 1$ per lane) were separated by cellulose acetate membrane SDS-PAGE (5\% acrylamide in concentration gel and $12 \%$ in separation gel) and analyzed by immunoblotting, as described previously $(13,14)$, using primary antibodies against LIMK1. Immunodetection was accomplished using an HRP-conjugated goat anti-rabbit secondary antibody (BS-13278, 1:1,000; Bioworld Technology, Inc., St. Louis Park, MN, USA) rotated 
for $1 \mathrm{~h}$ at room temperature. The protein bands were developed with a Novex ECL Chemiluminescent Substrate Reagent kit (Invitrogen; Thermo Fisher Scientific, Inc.) and analyzed by ImageJ software version 1.44 (National Institutes of Health, Bethesda, MD, USA).

Reverse transcription-polymerase chain reaction (RT-PCR). Total RNA from the cell lines was isolated using TRIzol reagent (Takara Bio, Inc., Otsu, Japan) and quantified using a NanoDrop ND-2000 (NanoDrop; Thermo Fisher Scientific, Inc., Wilmington, DE, USA), and reversely transcribed into cDNA using the EX Tag kit (TaKaRa, Japan) according to the manufacturer's protocols. RT-PCR was performed utilizing TaKaRa EX Taq DNA Polymerase (Takara Bio, Inc.) under the following conditions: Denaturation at $98^{\circ} \mathrm{C}$ for $10 \mathrm{sec}$, annealing at $54-62^{\circ} \mathrm{C}$ for $30 \mathrm{sec}$ and elongation at $72^{\circ} \mathrm{C}$ for $1 \mathrm{~min}$ for 20-30 cycles. The PCR products were visualized by electrophoresis on a 1.5\% agarose gel stained with GelRed ${ }^{\mathrm{TM}}$ (Biotium, Inc., Freemont, CA, USA). Images were obtained using the Gel Doc XR+ system (Bio-Rad Laboratories, Inc., Hercules, CA, USA) and analyzed using Image $\mathrm{Lab}^{\mathrm{TM}}$ software version 4.0 (Bio-Rad Laboratories, Inc.). The PCR primers used were: LIMK1, forward 5'-TGAGACAGGTGAGGTGAT GG-3' and reverse 5'-AGGCTGAGTCTTCTCGTCCA-3'; MDR1, forward 5'-ATATCAGCAGCCCACATCAT-3' and reverse 5'-GAAGCACTGGGATGTCCGGT-3'; and $\beta$-actin, forward 5'-CTGGGACGACATGGAGAAAA-3' and reverse 5'-AAGGAAGGCTGGAAGAGTGC-3'.

Plasmid transfections. To assess the effect of LIMK1 in the multidrug resistance, four different plasmids were transfected into MG63/VCR cells using Lipofectamine ${ }^{\mathrm{TM}} 2000$ transfection reagent (Invitrogen; Thermo Fisher Scientific, Inc.) according to the manufacturer's protocol. An expression plasmid coding for wild-type LIMK1-myc-enhanced cyan fluorescent protein (ECFP-LIMK1) and a short hairpin RNA (shRNA)-targeting LIMK1 construct, namely pSUPER-LIMK1-small interfering RNA (siRNA) (pSUPER-LIMK1), were constructed as described previously $(11,12)$. The pSUPER-negative control-siRNA (pSUPER) vector containing a scrambled non-target sequence and an empty vector containing myc-ECFP, were used as negative controls. Transfected cells were cultured for $24 \mathrm{~h}$ prior to being transferred into $60-\mathrm{mm}$ dishes or $96-$-well plates for subsequent experiments.

DOX efflux assay. Exponentially growing cells were plated onto $60-\mathrm{mm}$ Petri dishes and incubated with $1.5 \mu \mathrm{M}$ DOX for $3 \mathrm{~h}$ at $37^{\circ} \mathrm{C}$. Subsequently, cells were washed three times with ice-cold PBS, then centrifuged and suspended in ice-cold PBS and the mono-dispersed cells were analyzed by a flow cytometer (BD Biosciences, San Jose, CA, USA) using an argon laser of $15 \mathrm{~mW}$ at $488 \mathrm{~nm}$ (15). Cell fluorescence was evaluated in duplicate for each group, and all experiments were performed in triplicate.

Measurement of apoptosis. MG63/VCR cells in the exponential growth phase were plated onto $60-\mathrm{mm}$ Petri dishes and incubated with $500 \mathrm{ng} / \mathrm{ml} \mathrm{VCR}$ for $6 \mathrm{~h}$ at $37^{\circ} \mathrm{C}$. Upon incubation, apoptosis was evaluated using fluorescein
isothiocyanate-Annexin V and PI (BD Biosciences) according to the manufacturer's protocol. The analysis was performed in a flow cytometer (BD Biosciences) using an argon laser of $15 \mathrm{~mW}$ at $488 \mathrm{~nm}$. Cells ( $\geq 10,000$ cells/sample) were collected and analyzed using Cell Quest software version 7.5.3 (BD Biosciences).

Statistical analyses. All data are expressed as means \pm standard deviation. Statistical significance was assessed by the unpaired Student's t-test when comparing means between two groups or one-way analysis of variance with post-hoc Dunett's test to compare mean values among three or more groups. Statistical analysis was conducted with GraphPad Prism version 5.04 for Windows (GraphPad Software, Inc.). P $<0.05$ was considered to indicate a statistically significant difference.

\section{Results}

Elevated expression of LIMK1 in human osteosarcoma cells. To explore the role of LIMK1 in the progression of osteosarcoma, its expression in vitro was first assessed. Two osteosarcoma cell lines, U2OS and MG63, were used and compared with normal hFOB1.19 osteoblast cells. The expression of LIMK1 messenger RNA (mRNA) was significantly increased in both MG63 and U2OS cells compared with that in hFOB1.19 cells (by 2.6- and 4.2-fold, respectively) (Fig. 1A). A similar disparity was observed in western blot assays $(\mathrm{P}<0.01)$ (Fig. 1B). The expression of LIMK1 was also analyzed in human osteosarcoma tissues by immunohistochemistry. LIMK1 was highly expressed in the tumor parenchyma compared with that in the mesenchyme (Fig. 1C) in $83.3 \%$ of the specimens derived from 6 clinical patients. The expression of $\beta$-actin was unchanged between the parenchyma and mesenchyme. These results indicated that LIMK1 was overexpressed in osteosarcoma parenchyma, and suggested that LIMK1 may affect the progression of this type of cancer.

LIMK1 and MDR1 are overexpressed in multidrug resistant $M G 63 / V C R$ cells. In previous study (11), a multidrug resistant MG63/VCR subline was established by intermittent exposure of MG63 cells to gradually increasing VCR concentrations, as specified in the Materials and methods section. MG63/VCR cells were less sensitive to VCR-induced cytotoxicity than parental cells (Fig. 2A). The $\mathrm{IC}_{50}$ values of MG63 and MG63/VCR cells were 1.0 and $453.4 \mathrm{ng} / \mathrm{ml}$, respectively. MG63/VCR cells also exhibited cross-resistance to other structurally and mechanistically different drugs. The RI values of VCR, MTX, DOX, PTX and THP were 476.2, 4.7, 46.5, 379.0 and 23.7, respectively (Fig. 2B). To explore the mechanism by which MG63/VCR cells exhibited increased drug resistance, the expression of LIMK1 and MDR1/P-gp was examined by RT-PCR and western blot analysis. The expression of LIMK1 and MDR1 mRNA was elevated in MG63/VCR cells by 2.4- and 5.1-fold, respectively, compared with that in MG63 cells $(\mathrm{P}<0.01)$ (Fig. 2C). A similar result was obtained in the western blot assay, with increases by 4.7- and 6.6-fold in LIMK1 and MDR1 protein expression, respectively $(\mathrm{P}<0.01)$ (Fig. 2D). These results indicated that LIMK1 and MDR1/P-gp may be important in the MDR of osteosarcoma. 
A
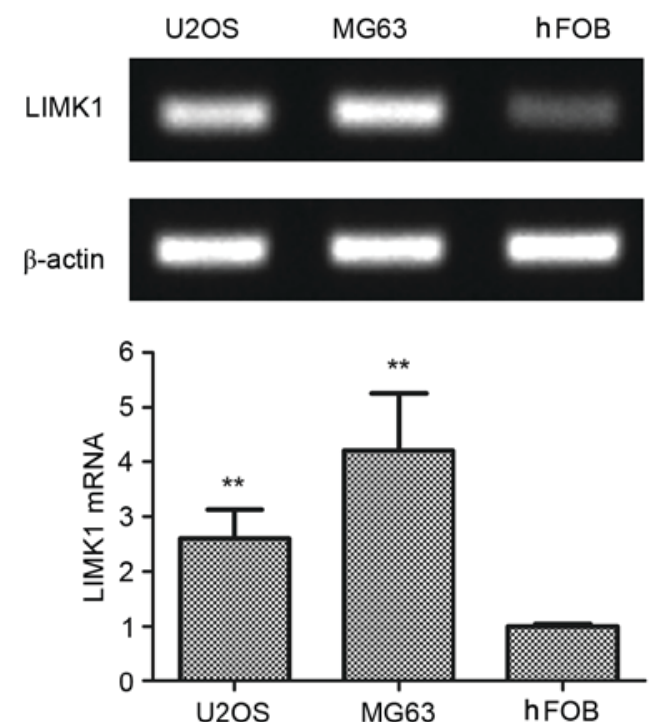

B

LIMK1

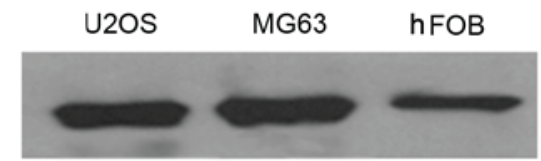

$\beta$-actin
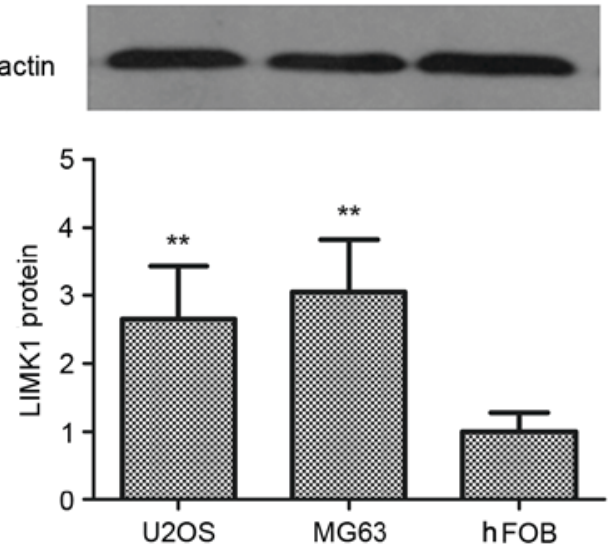

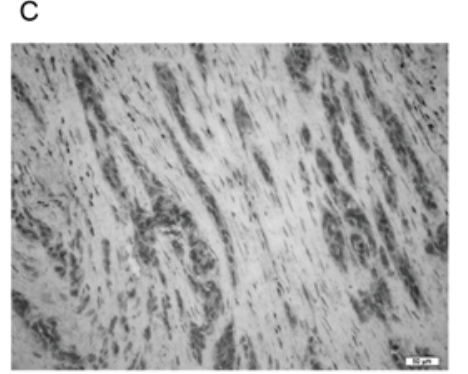

LIMK1 ( 200$)$

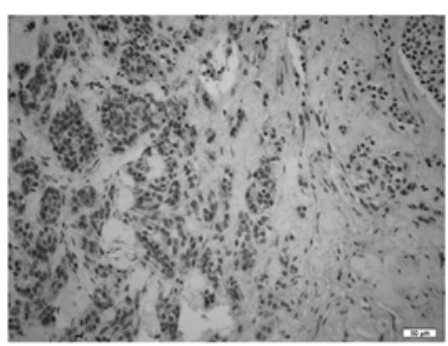

$\beta-\operatorname{actin}(\times 200)$

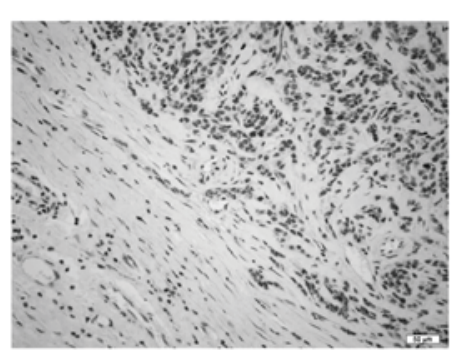

Negative control $(\times 200)$

Figure 1. Expression of LIMK1 in human osteosarcoma (U2OS and MG63) and osteoblast (hFOB) cells. (A) Reverse transcription-polymerase chain reaction and (B) western blotting were performed to detect the levels of LIMK1 mRNA and protein, respectively. The expression of LIMK1 was normalized to that of $\beta$-actin, and is shown in the bar graphs as means + standard deviation ( $\mathrm{n}=6 ;{ }^{* *} \mathrm{P}<0.01 \mathrm{vs.} \mathrm{hFOB}$ ). (C) Immunohistochemistry staining using antibodies against LIMK1 and $\beta$-actin in human osteosarcoma tissue. Tumor samples from a total of 6 patients were evaluated, and a representative image of the 5 positive results obtained is shown (scale bar, $50 \mu \mathrm{m}$ ). LIMK1, LIM kinase 1; mRNA, messenger RNA; hFOB, human fetal osteoblasts.

A

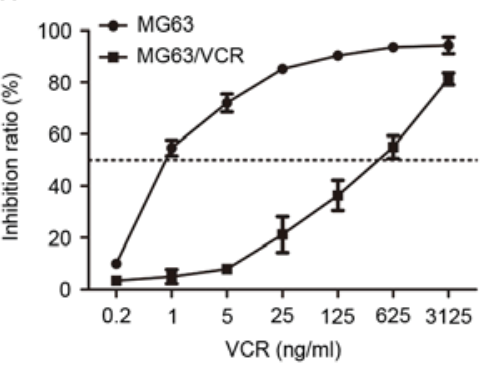

\begin{tabular}{lccc}
\hline \multirow{2}{*}{ Reagents } & \multicolumn{2}{c}{ IC50 $(\mathrm{ng} / \mathrm{ml})$} & \\
\cline { 2 - 3 } & MG63 & MG63/VCR & \\
\hline VCR & 0.9521 & 453.4 & 476.2 \\
MTX & 25.39 & 120.4 & 4.742 \\
DOX & 289.8 & 13460 & 46.45 \\
PTX & 1.24 & 470.3 & 379.3 \\
THP & 1.691 & 40.06 & 23.69 \\
\hline
\end{tabular}

B
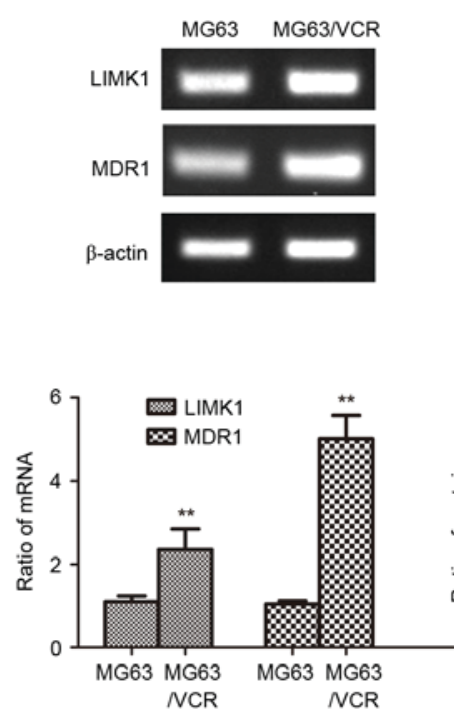

c
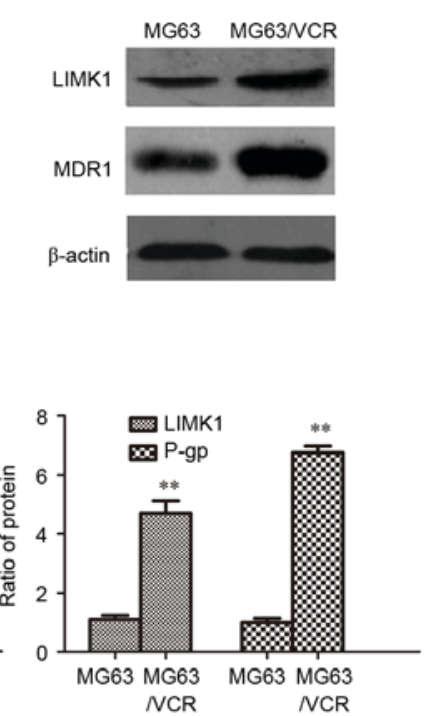

Figure 2. Expression of LIMK1 and MDR1 in MG63 and MG63/VCR cells. (A) Dose-dependent cell viability curves of cells treated with VCR. (B) Agarose gel electrophoresis of the reverse transcription-polymerase chain reaction products for LIMK1 and MDR1. (C) Western blotting was performed to detect the protein levels of LIMK1 and MDR1/P-gp proteins. (D) The concentration of each drug that produced 50\% inhibition of growth $\left(\mathrm{IC}_{50}\right)$ and the $\mathrm{RI}$ values of various commonly used anticancer drugs in MG63 and MG63/VCR cells are shown. ${ }^{* *} \mathrm{P}<0.01 \mathrm{vs.} \mathrm{MG63} \mathrm{using} \mathrm{the} \mathrm{Student's} \mathrm{t-test.} \mathrm{All} \mathrm{results} \mathrm{are} \mathrm{from}$ three or four independent experiments. Data are expressed as means \pm standard deviation. LIMK1, LIM kinase 1; MDR1, multidrug resistance protein 1; P-gp, P-glycoprotein; mRNA, messenger RNA; $\mathrm{IC}_{50}, 50 \%$ inhibitory concentration; RI, resistance index; MG63/VCR, multidrug resistant MG63 cells; VCR, vincristine; MTX, methotrexate; DOX, doxorubicin; PTX, paclitaxel; THP, pirarubicin. 

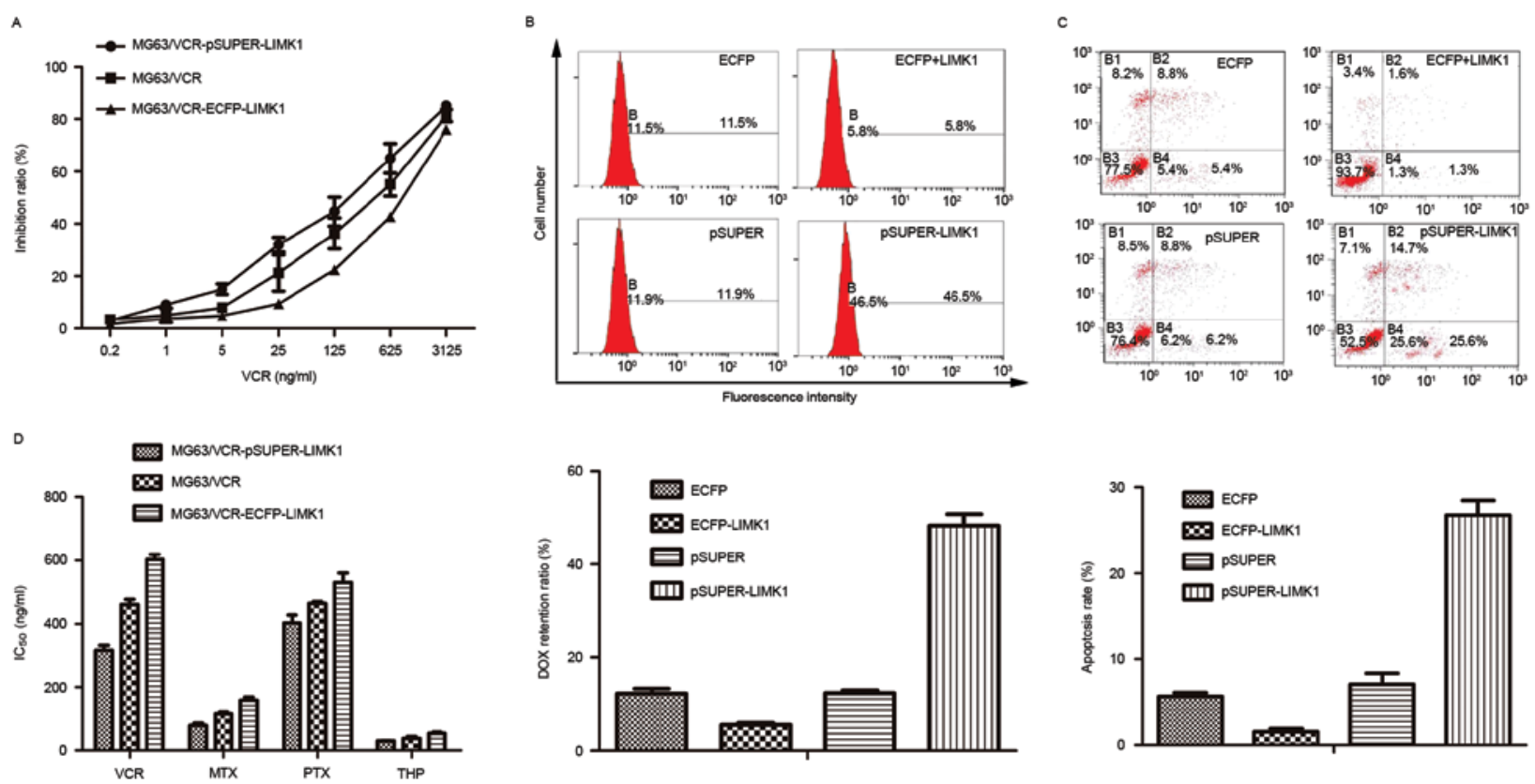

Figure 3. LIMK1 is critical for drug resistance in osteosarcoma cells. (A) MG63/VCR, MG63/VCR pSUPER-LIMK1-transfected and MG63/VCR ECFP-LIMK1-transfected cells were treated with different concentrations of VCR, and the cell viability was analyzed using the Cell Counting kit-8 assay. (B) The fluorescence intensity of DOX was analyzed quantitatively by flow cytometry. The mean fluorescence intensity + SD is shown in the bar graph. (C) Cell lines were incubated with $\mathrm{VCR}\left(\mathrm{IC}_{50}\right)$ and assessed for early apoptosis by Annexin V/propidium iodide staining and flow cytometry analysis. (D) The concentration at which each drug produced $50 \%$ inhibition of growth $\left(\mathrm{IC}_{50}\right)$ is shown in the histogram. Representative data are shown. The histogram represents means \pm SD of five independent experiments. LIMK1, LIM kinase 1; MG63/VCR, multidrug resistant MG63 cells; ECFP, enhanced cyan fluorescent protein; $\mathrm{IC}_{50}, 50 \%$ inhibitory concentration; VCR, vincristine; MTX, methotrexate; DOX, doxorubicin; PTX, paclitaxel; THP, pirarubicin; SD, standard deviation

LIMK1 serves a key role in the MDR of MG63/VCR cells. To assess whether LIMK1 was involved in the MDR of MG63/VCR cells, LIMK1 was knocked down by transfecting these cells with pSUPER-LIMK1 plasmids coding LIMK1 shRNA target sequences. In addition, LIMK1 expression was upregulated by transfecting the cells with ECFP-LIMK1 plasmids coding wild-type LIMK1. The viability of the transfected cells was evaluated upon treatment with a series of diluted drugs. A representative result with VCR is shown in Fig. 3A. The $\mathrm{IC}_{50}$ values for VCR, MTX, PTX and THP were calculated and shown in Fig. 3B. Overall, cells expressing high levels of LIMK1 exhibited strong resistance to all chemotherapeutic drugs. DOX is a commonly used anticancer drug that has autofluorescence at the same wavelength as PI (15). The concentration of DOX was evaluated in cells transfected with different plasmids. The results revealed that DOX accumulation was the highest in MG63/VCR cells transfected with pSUPER-LIMK1 compared with in other groups, as evidenced by the increased DOX fluorescence intensity in these cells. This suggested that DOX efflux was downregulated along with LIMK1. By contrast, cells transfected with the ECFP-LIMK1 plasmid exhibited an increased efflux ability and reduced sensitivity to the drugs tested $(\mathrm{P}<0.01)$ (Fig. 3C). The results from the apoptosis assays were consistent with these findings. Cytometric dot-plot images of MG63/VCR cells obtained after $6 \mathrm{~h}$ of incubation with the $\mathrm{IC}_{50}$ of VCR were used to evaluate the population of apoptotic cells in the lower right quadrant of the graphs (which corresponds to Annexin V-positive and PI-negative cells) (Fig. 3D). The apoptosis rate in cells transfected with the pSUPER-LIMK1 plasmid $(25.6 \%)$ was higher than that in the empty vector group (6.2\%) and in the cells transfected with the ECFP-LIMK1 plasmid $(1.3 \%)(\mathrm{P}<0.01)$ (Fig. 3D). These results indicate that LIMK1 is important in the MDR of MG63/VCR cells.

LIMK1 functions through regulating the expression of MDRI/ $P$ - $g p$. LIMK1 and MDR1/P-gp were expressed at higher levels in MG63/VCR cells than in MG63 cells (Fig. 2). To explore the connection between the two genes, the expression of LIMK1 and MDR1/P-gp was assessed in MG63/VCR cells following transfection with LIMK1 shRNA, wild-type LIMK1 or negative control vector. The results indicated that both LIMK1 mRNA and protein expression levels were increased in MG63/VCR cells following transfection with wild-type LIMK1, compared with those in cells transfected with the empty vector. LIMK1 mRNA and protein expression levels were significantly reduced in cells transfected with LIMK1 shRNA, compared with those in cells transfected with the empty vector or wild-type LIMK1. Notably, the change in MDR1 expression was completely consistent with that in LIMK1 expression $(\mathrm{P}<0.05)$ (Fig. 4A and B). Immunocytochemistry staining was performed to compare the expression of MDR1 in the same cells. The results demonstrated that MDR1/P-gp expression was increased significantly in MG63/VCR cells transfected with ECFP-LIMK1 plasmid, while transfection with LIMK1 shRNA decreased MDR1/P-gp expression (Fig. 4C). These data suggest that LIMK1 may affect MDR by regulating the expression of MDR1/P-gp.

\section{Discussion}

In the present study, a novel function of LIMK1 in regulating MDR was identified. Previous studies demonstrated that 

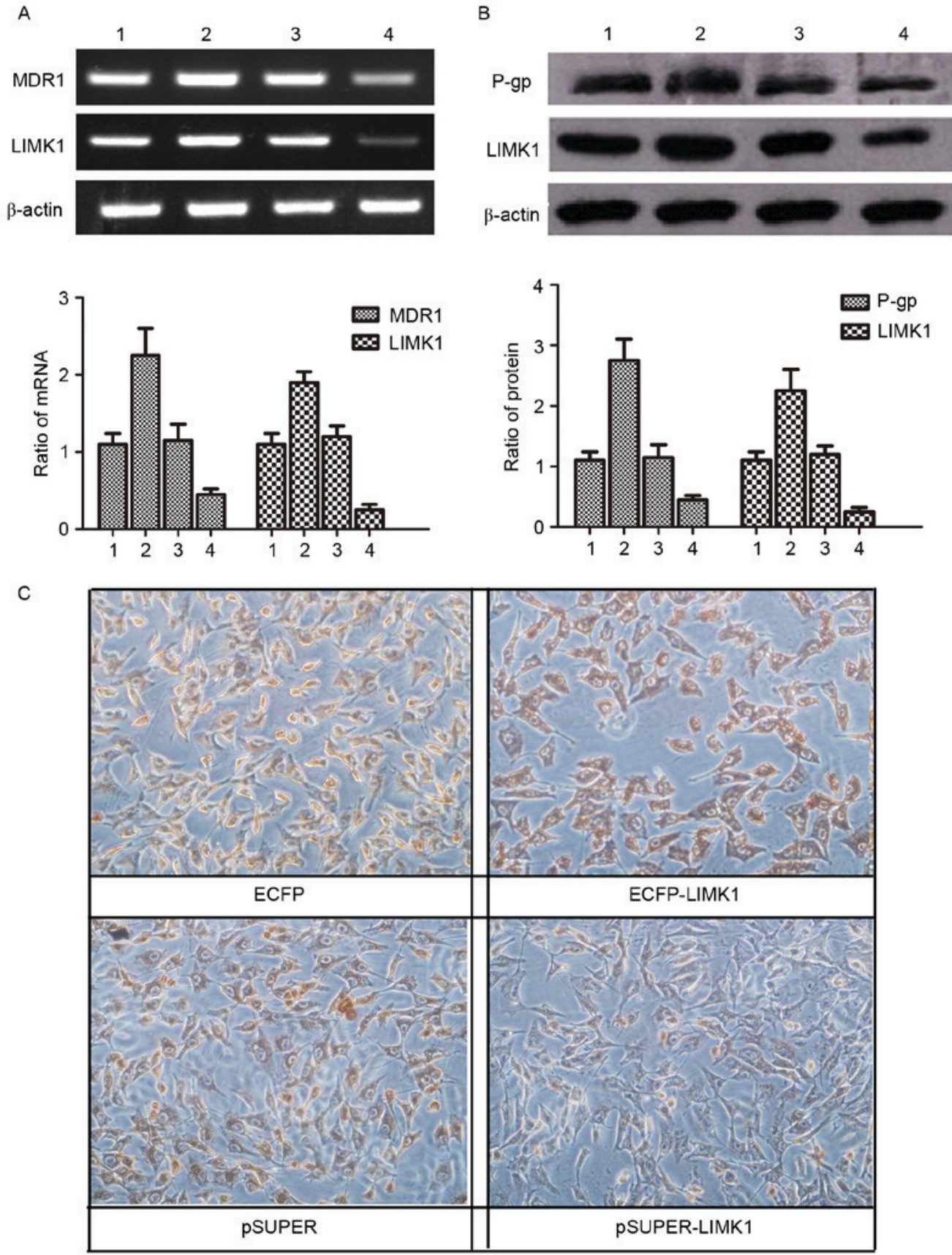

Figure 4. LIMK1 functions through regulating the expression of MDR1/P-gp. (A and B) Lanes 1, 2, 3 and 4 represent MG63/VCR cells transfected with plasmids for ECFP, ECFP-LIMK1, pSUPER and pSUPER-LIMK1 expression, respectively. (A) Agarose gel electrophoresis of the reverse transcription-polymerase chain reaction products for LIMK1 and MDR1 in MG63/VCR cells transfected with different plasmids. (B) Western blot analysis of LIMK1 and MDR1/P-gp proteins in MG63/VCR cells transfected with different plasmids. (C) Immunohistochemistry staining using antibodies against MDR1 in MG63 and MG63/VCR cells transfected with LIMK1 short hairpin RNA (pSUPER-LIMK1) or a control pSUPER-negative control-small interfering RNA vector (magnification, x200; scale bar, $50 \mu \mathrm{m}$ ). LIMK1, LIM kinase 1; MG63/VCR, multidrug resistant MG63 cells; mRNA, messenger RNA; MDR1, multidrug resistance protein 1; P-gp, P-glycoprotein; ECFP, enhanced cyan fluorescent protein.

LIMK1 was overexpressed and highly active in cells and tissues of certain malignant tumors, including prostate and breast cancer $(16,17)$. LIMK1 was considered to be a key molecule that stimulated malignant tumor cell invasion and metastasis, or possibly a new 'oncogene' $(18,19)$. However, few studies linked LIMK1 with MDR, which represents a major challenge for managing the treatment of the majority cancers (20). In the present study, the expression of LIMK1 mRNA and protein in twohuman osteosarcoma cell lines (MG63 and U2OS) was markedly higher than that in hFOB cells (Fig. 1A and B). Furthermore, the expression of LIMK1 in tumor parenchyma cells was significantly higher compared with that in mesenchymal cells (Fig. 1C), suggesting that LIMK1 was responsible for the genesis and development of osteosarcoma.

A multidrug resistant MG63/VCR subline was established by intermittent exposure of MG63 cells to VCR. This subline exhibited cross-resistance to other commonly used drugs to treat osteosarcoma, but which are structurally and mechanistically different to VCR (Fig. 2B) (1,21). These drugs included 
an anti-microtubule agent (PTX), an antimetabolite (MTX) and two topoisomerase II inhibitors (DOX and THP) (22). Notably, the mRNA and protein expression levels of LIMK1 and MDR1/P-gp were higher in MG63/VCR cells than in MG63 cells (Fig. 2C and D). Therefore, it was hypothesized that there was a correlation between LIMK1 and MDR in osteosarcoma.

In order to verify this conjecture, additional experiments were conducted by upregulating and downregulating LIMK1 in MG63/VCR cells. Downregulation of LIMK1 inhibited the efflux of DOX and decreased the MDR of these cells compared with that of the controls. In addition, the sensitivity of these cells to VCR was elevated (Fig. 3). Of note, the expression of MDR1/P-gp, which was also increased in MG63/VCR cells compared with that in MG63 cells, was changed in accordance with the expression of LIMK1. MDR1 belongs to the ABC transporter family that regulates the efflux across the plasma membrane of multiple structurally and mechanistically unrelated chemotherapeutic agents (3). MDR1/P-gp-mediated MDR has been long considered a classical mechanism of MDR in malignant tumors, including osteosarcoma $(3,20)$. The expression of MDR1 in MG63/VCR cells was increased significantly in cells overexpressing LIMK1 compared with that in cells transfected with empty vector. In addition, downregulation of LIMK1 resulted in a significant decrease in the expression of MDR1. These results indicate that LIMK1 serves an important role in MDR through regulating the expression of MDR1/P-gp. The RhoA/LIMK1/cofilin signaling pathway may have certain connection with the $\mathrm{ABC}$ transporter family. However, understanding the precise molecular mechanism requires further investigation.

In summary, the overexpression of LIMK1 increases MDR1/P-gp expression and has major effects on the MDR of human osteosarcoma. The present study provides new insights into the function of LIMK1 and suggests that altering LIMK1 is a potential new therapeutic strategy for osteosarcoma.

\section{Acknowledgements}

The present study was supported by Projects of International Cooperation of Jilin Provincial Science \& Technology Department (grant no. 20150101175JC) and the National Natural Science Foundation of China (grant nos. 81172000 and 30772488).

\section{References}

1. Luetke A, Meyers PA, Lewis I and Juergens H: Osteosarcoma treatment-where do we stand? A state of the art review. Cancer Treat Rev 40: 523-532, 2014.

2. Ottaviani G and Jaffe N: The epidemiology of osteosarcoma. Cancer Treat Res 152: 3-13, 2009.

3. Holohan C, Van Schaeybroeck S, Longley DB and Johnston PG: Cancer drug resistance: An evolving paradigm. Nat Rev Cancer 13: 714-726, 2013.

4. Thomas $\mathrm{H}$ and Coley HM: Overcoming multidrug resistance in cancer: An update on the clinical strategy of inhibiting p-glycoprotein. Cancer Control 10: 159-165, 2003.

5. Maekawa M, Ishizaki T, Boku S, Watanabe N, Fujita A, Iwamatsu A, Obinata T, Ohashi K, Mizuno K and Narumiya S: Signaling from Rho to the actin cytoskeleton through protein kinases ROCK and LIM-kinase. Science 285: 895-898, 1999.
6. Ohashi K, Nagata K, Maekawa M, Ishizaki T, Narumiya S and Mizuno K: Rho-associated kinase ROCK activates LIM-kinase 1 by phosphorylation at threonine 508 within the activation loop. J Biol Chem 275: 3577-3582, 2000

7. Arber S, Barbayannis FA, Hanser H, Schneider C, Stanyon CA, Bernard $\mathrm{O}$ and Caroni $\mathrm{P}$ : Regulation of actin dynamics through phosphorylation of cofilin by LIM-kinase. Nature 393: 805-809, 1998.

8. Yang N, Higuchi O, Ohashi K, Nagata K, Wada A, Kangawa K, Nishida E and Mizuno K: Cofilin phosphorylation by LIM-kinase 1 and its role in Rac-mediated actin reorganization. Nature 393: 809-812, 1998.

9. Li R, Doherty J, Antonipillai J, Chen S, Devlin M, Visser K, Baell J, Street I, Anderson RL and Bernard O: LIM kinase inhibition reduces breast cancer growth and invasiveness but systemic inhibition does not reduce metastasis in mice. Clin Exp Metastasis 30: 483-495, 2013.

10. Chen Q, Jiao D, Hu H, Song J, Yan J, Wu L and Xu LQ: Downregulation of LIMK1 level inhibits migration of lung cancer cells and enhances sensitivity to chemotherapy drugs. Oncol Res 20: 491-498, 2013.

11. Zhang H, Wang Y, Xing F, Wang J, Wang Y, Wang H, Yang Y and Gao Z: Overexpression of LIMK1 promotes migration ability of multidrug-resistant osteosarcoma cells. Oncol Res 19: 501-509, 2011.

12. Zhang HS, Zhao JW, Wang H, Zhang HY, Ji QY, Meng LJ, Xing FJ, Yang ST and Wang Y: LIM kinase 1 is required for insulin-dependent cell growth of osteosarcoma cell lines. Mol Med Rep 9: 103-108, 2014.

13. Takemura M, Mishima T, Wang Y, Kasahara J, Fukunaga K, Ohashi $\mathrm{K}$ and Mizuno $\mathrm{K}: \mathrm{Ca}^{2+} / \mathrm{calmodulin}$-dependent protein kinase IV-mediated LIM kinase activation is critical for calcium signal-induced neurite outgrowth. J Biol Chem 284: 28554-28562, 2009.

14. Zhao JW, Zhang MR, Ji QY, Xing FJ, Meng LJ and Wang Y: The role of slingshot-1L (SSH1L) in the differentiation of human bone marrow mesenchymal stem cells into cardiomyocyte-like cells. Molecules 17: 14975-14994, 2012.

15. Zhang X, Peng X, Yu W, Hou S, Zhao Y, Zhang Z, Huang X and Wu K: Alpha-tocopheryl succinate enhances doxorubicin-induced apoptosis in human gastric cancer cells via promotion of doxorubicin influx and suppression of doxorubicin efflux. Cancer Lett 307: 174-181, 2011.

16. Davila M, Frost AR, Grizzle WE and Chakrabarti R: LIM kinase 1 is essential for the invasive growth of prostate epithelial cells: Implications in prostate cancer. J Biol Chem 278: 36868-36875, 2003.

17. Tapia T, Ottman R and Chakrabarti R: LIM kinase1 modulates function of membrane type matrix metalloproteinase 1: Implication in invasion of prostate cancer cells. Mol Cancer 10: 6, 2011.

18. Huang TY, DerMardirossian C and Bokoch GM: Cofilin phosphatases and regulation of actin dynamics. Curr Opin Cell Biol 18: 26-31, 2006.

19. Guo H, Wu F, Wang Y, Yan C and Su W: Overexpressed ubiquitin ligase Cullin7 in breast cancer promotes cell proliferation and invasion via down-regulating p53. Biochem Biophys Res Commun 450: 1370-1376, 2014.

20. Kathawala RJ, Gupta P, Ashby CR Jr and Chen ZS: The modulation of ABC transporter-mediated multidrug resistance in cancer: A review of the past decade. Drug Resist Updat 18: 1-17, 2015.

21. Ta HT, Dass CR, Choong PF and Dunstan DE: Osteosarcoma treatment: State of the art. Cancer Metastasis Rev 28: 247-263, 2009.

22. Yang JZ, Ma SR, Rong XL, Zhu MJ, Ji QY, Meng LJ, Gao YY, Yang YD and Wang Y: Characterization of multidrug-resistant osteosarcoma sublines and the molecular mechanisms of resistance. Mol Med Rep 14: 3269-3276, 2016.

This work is licensed under a Creative Commons Attribution-NonCommercial-NoDerivatives 4.0 International (CC BY-NC-ND 4.0) License. 